\title{
IMPACTO DA HIPERTENSÃO ARTERIAL NA QUALIDADE DE VIDA DE IDOSOS RESIDENTES NA ZONA RURAL
}

\section{IMPACT OF HYPERTENSION ON QUALITY OF LIFE OF ELDERLY IN RURAL AREA}

\section{IMPACTO DE LA HIPERTENSÓN ARTERIAL EN LA CALIDAD DE VIDA DE LOS RESIDENTES MAYORES EM AREA RURAL}

Mariana Mapelli de Paiva ${ }^{1}$, Flavia Aparecida Dias ${ }^{2}$, Nayara Paula Fernandes Martins Molina ${ }^{3}$, Darlene Mara dos Santos Tavares ${ }^{4}$

\section{RESUMO}

Objetivou descrever as variáveis relacionadas com a hipertensão arterial sistêmica (HAS) e comparar com os escores de qualidade de vida (QV) entre idosos com e sem HAS da zona rural de Uberaba - MG. Foram constituídos dois grupos: idosos com HAS (353) e sem HAS (353). Utilizaram-se os instrumentos: estruturado, World Health Organization Quality of Life - BREF (WHOQOL-BREF), World Health Organization Quality of Life Assessment for Older Persons (WHOQOL-OLD). Realizou análise descritiva e teste $t$-Student $(p<0,05)$. A maioria dos idosos foi diagnosticada há menos de 10 anos, por meio de exame médico de rotina, aferiam a pressão arterial, usavam medicamento e consumiam pouco sal. Os idosos com HAS apresentaram menor escore de QV em relação aos sem HAS. São necessárias medidas que minimize os impactos que a HAS causa na QV.

Descritores: Hipertensão, Qualidade de Vida, Idoso, Enfermagem, Enfermagem Geriátrica.

\begin{abstract}
Aimed to describe the variables related to arterial hypertension (SAH) and compare with the scores of quality of life (QOL) among elderly people with and without hypertension in rural area of Uberaba, MG. Were comprised two groups: elderly patients with SAH (353) and without hypertension (353). We used the following instruments: structured, World Health Organization Quality of Life - BREF (WHOQOL-BREF), World Health Organization Quality of Life Assessment for Older Persons (WHOQOL-OLD). Held descriptive analysis and Student's t-test $(\mathrm{p}<0.05)$. The elderly with hypertension had been diagnosed for less than 10 years, through medical examination of routine, hip circumferences were used blood pressure, used medication and drank little salt. The elderly with hypertension had lower scores quality of life in relation to those without hypertension. It is necessary to take measures to minimize the impacts that cause high blood pressure in the QOL.

Descriptores: Hypertension, Quality of life, Elderly, Nursing, Geriatric Nursing.

\footnotetext{
${ }^{1}$ Enfermeira. Mestre em Atenção à Saúde. Doutoranda do Programa de Pós-Graduação stricto sensu em Saúde Coletiva.

${ }^{2}$ Enfermeira. Mestre em Atenção à Saúde. Doutoranda do Programa de Pós-Graduação stricto sensu em Atenção à Saúde.

${ }^{3}$ Enfermeira. Mestre em Atenção à Saúde. Doutoranda do Programa de Pós-Graduação stricto sensu em Atenção à Saúde.

${ }^{4}$ Enfermeira. Doutor em Enfermagem. Professora Associada do Departamento de Enfermagem em Educação e Saúde

Comunitária do Curso de Graduação em Enfermagem. Instituto de Ciências da Saúde. Universidade Federal do Triângulo Mineiro.
} 


\section{RESUMEN}

Tiene como objetivo describir las variables relacionadas con la hipertensión arterial sistêmica (HAS) y comparar con las puntuaciones de calidad de vida (QOL), entre los adultos mayores con y sin hipertensión en el área rural de Uberaba, MG. Estaban compuestas por dos grupos: los pacientes ancinos com hipertensión arterial (353) y sin hipertensión (353). Hemos utilizado los siguientes instrumentos: estructura, Organización Mundial de la Salud Calidad de Vida - BREF (WHOQOL-BREF), Organización Mundial de la Salud Evaluación de la calidad de vida de las Personas de Edad (WHOQOL-OLD). Realizó análisis descriptivo y la prueba t de Student ( $\mathrm{p}<0.05$ ). La mayoría de las personas de edad avanzada fue diagnosticado de menos de 10 años, mediante examen médico de rutina, se utilizaron las circunferencias cadera presión sanguínea, medicamento que se usa y bebió un poco de sal. Las personas de edad avanzada con hipertensión había puntuaciones más bajas de la $\mathrm{CV}$ en relación con aquellas personas sin hipertensión. Las medidas que sean necesarias para minimizar los impactos que la HAS en la CV.

Descriptores: Hipertensión, Calidad de vida, Anciano, Enfermería, Enfermería Geríatrica.

\section{INTRODUÇÃO}

O processo de transição demográfica tem trazido à necessidade de se repensar a dimensão dos serviços necessários para as próximas décadas, e o aumento do contingente de idosos acarreta no aumento da carga de doenças, em destaque as doenças crônicas não transmissíveis (DCNT). ${ }^{1}$

Tais doenças podem apresentar peculiaridades relacionadas ao espaço rural, dadas as possíveis dificuldades no diagnóstico, adesão ao tratamento e evolução das comorbidades e complicações. Dentre estas, destaca-se que a hipertensão arterial sistêmica (HAS) que atinge, no Brasil, aproximadamente, 65,7\% dos idosos segundo a Vigilância de Fatores de Risco e Proteção para doenças crônicas por inquérito telefônico. ${ }^{2}$

A HAS é caracterizada como uma condição multifatorial que apresenta níveis elevados de pressão arterial (PA), considerando a PA sistólica maior ou igual a $140 \mathrm{mmHg}$ e/ou PA diastólica maior ou igual a 90 mmHg. ${ }^{3}$ Esta condição pode ocasionar complicações no coração, encéfalo, rins e vasos sanguíneos ${ }^{3}$, e de acordo com pesquisa realizada com idosos hipertensos, estes tiveram pior qualidade de vida $(\mathrm{QV})$ em aspectos relacionadas a esta. $^{4}$

O conceito de QV adotado para o presente estudo será o de um grupo de estudiosos, apoiados pela Organização Mundial de Saúde (OMS), que a define como "percepção do indivíduo de sua posição na vida no contexto da cultura e 
sistema de valores nos quais ele vive e em relação aos seus objetivos, expectativas, padrões e preocupações". 5:1405

Considerando a alta prevalência de HAS entre os idosos, seu impacto na QV e a escassez de pesquisas brasileiras conduzidas na área rural brasileira que avaliam este aspecto, utilizando instrumentos específicos para esta faixa etária propôs-se a desenvolver este estudo. Assim, tem-se o intuito de contribuir para ampliar o conhecimento sobre esta temática, de maneira a subsidiar a formulação de ações e políticas públicas para os idosos com HAS residentes na área rural.

Os objetivos delineados foram: descrever as características sociodemográficas e número de morbidades autorreferidas dos idosos com e sem HAS; descrever as variáveis relacionadas à HAS; e comparar os escores de QV entre os idosos com e sem HAS residentes na área rural.

\section{MÉTODOS}

Esta pesquisa se caracteriza como inquérito domiciliar, transversal e observacional, desenvolvido na área rural do município de Uberaba-Minas Gerais (MG), Brasil.

$$
\text { Participaram da presente }
$$
investigação pessoas com 60 anos ou mais de idade, que residiam na área rural do referido município e sem declínio cognitivo. A HAS foi identificada pela referência dos idosos. Foram constituídos dois grupos, pareados segundo sexo e faixa etária: 353 idosos com HAS e 353 sem HAS.

Utilizaram-se os instrumentos: Mini Exame de Estado Mental (MEEM) ${ }^{6}$, para avaliar o declínio cognitivo, critério de exclusão da presente pesquisa; instrumento estruturado, baseado no questionário Older Americans Resources and Services $(\mathrm{OARS})^{7}$, para a caracterização da população; instrumento construído pelos pesquisadores contendo as variáveis relacionadas à HAS; World Health Organization Quality of Life - BREF (WHOQOL-BREF) $^{8}$ e World Health Organization Quality of Life Assessment for Older Persons (WHOQOL-OLD) ${ }^{9}$ para mensurar a QV.

Estudaram-se as variáveis sociodemográficas (sexo, faixa etária, estado conjugal, escolaridade, renda individual, arranjo domiciliar, número de morbidades); relacionadas a HAS (tempo 
de diagnóstico, como descobriu a HAS, uso de medicamentos, interrupção do tratamento, hábito de aferir a PA e quantidade de sal nas refeições), domínios da QV (físico, psicológico, relações sociais e meio ambiente) e facetas da QV (funcionamento dos sentidos, autonomia, atividades passadas, presentes e futuras, participação social, morte e morrer e intimidade).

Os dados foram coletados no domicílio por 14 entrevistadores devidamente treinados no período de junho de 2010 a março de 2011. Foi criada uma planilha no programa Excel $^{\circledR}$, e os dados processados em microcomputador, em dupla entrada. Posteriormente, verificou-se a consistência entre os dois bancos de dados, procedendo às correções pertinentes pela entrevista original.

Para a realização da análise, os dados foram transportados para o software Statistical Package for the Social Sciences (SPSS) versão 17.0. Os domínios do WHOQOL-BREF e facetas do WHOQOLOLD foram analisados isoladamente com as respectivas sintaxes. Procedeu-se análise descritiva por meio de distribuição de frequências absolutas e percentuais; média e desvio padrão. Para comparar os escores de QV entre os idosos utilizou-se o teste $t$ Student $(p<0,05)$.

O projeto foi aprovado pelo Comitê de Ética em Pesquisa com Seres Humanos da Universidade Federal do Triângulo Mineiro, $n^{0} 1477$. Somente após a anuência do entrevistado e assinatura do Termo de Consentimento Livre e Esclarecido, conduziu-se a entrevista.

\section{RESULTADOS}

A maioria dos idosos era do sexo masculino $(57,2 \%)$ e faixa etária de $60+70$ $\operatorname{anos}(64 \%)$.

Em ambos os grupos, prevaleceram aqueles que moravam com esposo (a) ou companheiro (a) $(65,7 \%$ com HAS, $71,1 \%$ sem HAS), com 4 -8 anos de estudo (35, $1 \%$ com HAS, $38,8 \%$ sem HAS), renda de um salário mínimo (46,2\% com HAS, $46 \%$ sem HAS), residiam somente com o cônjuge $(45,6 \%$ com HAS, 49,9\% sem HAS). Os idosos com HAS $(39,7 \%)$ referiram maior número de doenças (4 a 7) em relação aos sem HAS $(47,6 \%)$ que apresentavam 1 a 4.

Entre os idosos com HAS prevaleceram aqueles que tinham a doença há menos de 10 anos (70,3\%), tiveram o diagnóstico em exame médico de rotina 
(44,3\%), tinham o hábito de aferir a PA (73,5\%), faziam uso regular de medicamentos $(95,7 \%)$, não interromperam o tratamento nenhuma vez $(77,5 \%)$ e utilizavam pouca quantidade sal nas refeições $(90,3 \%)$.

Tanto a maioria dos idosos com HAS $(57,2 \%)$ como os sem HAS $(61,8 \%)$ avaliaram a QV como boa. O mesmo se avaliou em relação à satisfação com a saúde, expressa pelos idosos com HAS $(60,6 \%)$ e sem HAS $(60,3 \%)$.

Os maiores escores de QV estiveram no domínio relações sociais e na faceta intimidade; enquanto os menores foram no meio ambiente e participação social, para ambos os grupos (Tabela 1).

Os idosos com HAS apresentaram menor escore de QV nos domínios: físico $(p=0,001)$, psicológico $(p=0,020)$ e meio ambiente $(p=0,015)$; e nas facetas: funcionamento dos sentidos $(p=0,002)$, autonomia $(p=0,023)$, participação social $(p=0,001)$ e intimidade $(p=0,004)$ quando comparados aqueles sem HAS (Tabela 1).

Tabela 1. Distribuição dos escores de QV dos idosos com e sem HAS. Uberaba, 2011.

\begin{tabular}{llll}
\hline Variáveis & Com & \multicolumn{2}{l}{ Sem } \\
\cline { 2 - 3 } & Méd Desv & Méd Desv & $p$ \\
\hline
\end{tabular}

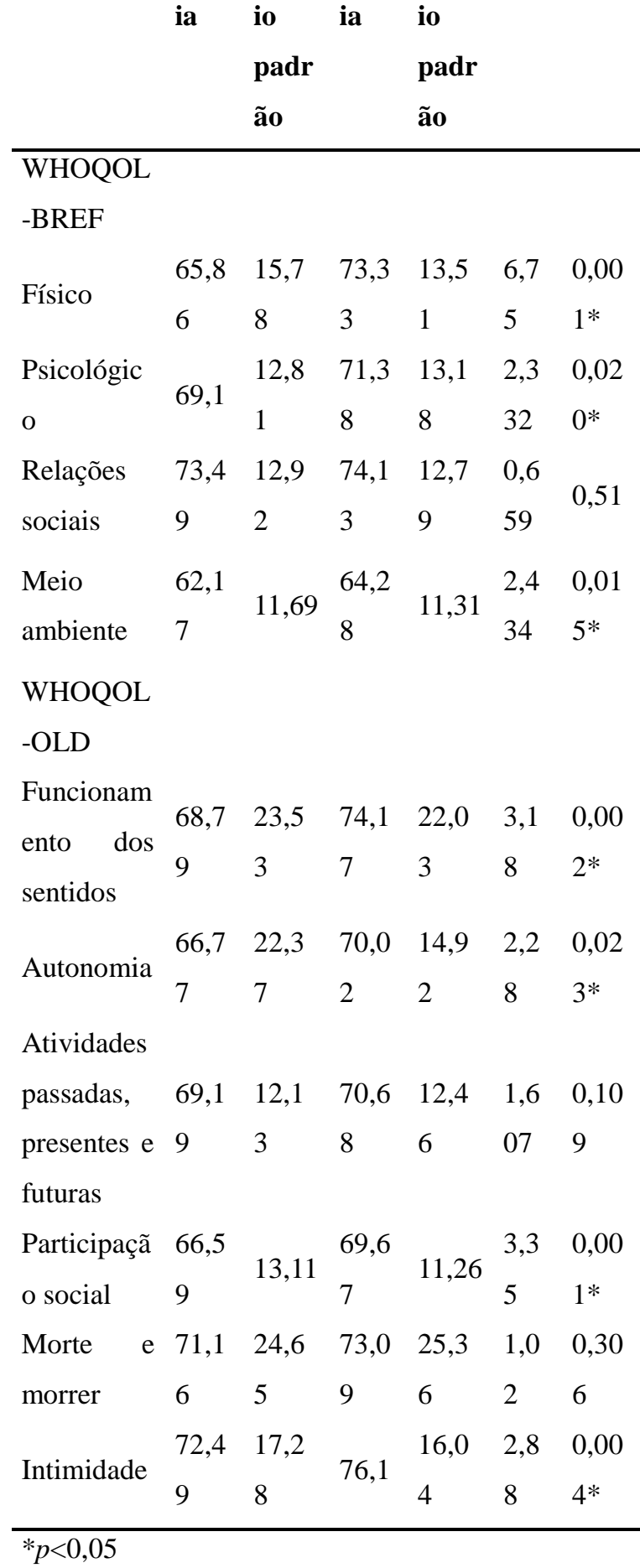

\section{DISCUSSÃO}


As características sociodemográficas dos idosos com HAS foram semelhantes aos sem HAS. Na comparação com a literatura científica observaram-se resultados divergentes, em estudos nacionais com adultos e idosos da área rural, em relação ao sexo, em que prevaleceu o feminino ${ }^{10,11}$ e semelhantes quanto a faixa etária ${ }^{10,11}$ e estado conjugal. $^{10,11}$ Quanto à escolaridade ${ }^{10}$, renda $^{11}$ e arranjo de moradia ${ }^{11}$ os achados diferem de pesquisas com adultos e idosos com e sem HAS das áreas urbanas e rurais. Neste contexto reforça a necessidade do enfermeiro obter estes dados e gerar informações para subsidiar o cuidado de enfermagem, considerando a extensão territorial do Brasil e suas diferenças regionais. Desta forma, o planejamento do cuidado de enfermagem gerontológica deve atender as peculiaridades do idoso a ser atendido.

Quanto ao número de morbidades percentual superior de idosos sem HAS $(72,5 \%)$ apresentaram menos de quatro comorbidades em pesquisa realizada na área urbana e rural de Teixeiras-MG. ${ }^{11}$ Considerando que os idosos com HAS apresentaram maior número de doenças, o que poderá acarretar maior comprometimento das condições de saúde, o enfermeiro por meio do pensamento crítico $^{12}$ deve identificar tais comorbidades e propor ações preventivas. Cabe destacar que as atividades promocionais em saúde também contribuem para a melhoria da saúde em geral além da maior autonomia no processo decisório e autocuidado.

O tempo de diagnóstico de HAS foi inferior ao obtido em investigação realizada com adultos e idosos da área urbana da região oeste de São Paulo-SP, que tinham a doença há mais de cinco anos. $^{13}$ Os idosos da presente pesquisa descobriram a HAS em consulta de rotina; este fato pode estar relacionado a característica assintomática da doença. Ressalta-se que isto pode influenciar no tratamento da HAS devido comportamentos supersticiosos como observado em inquérito com idosos rurais em Minas Gerais. ${ }^{14}$ Estes achados expressam as diferenças na organização dos diversos serviços de saúde brasileiros e também denotam a necessidade do enfermeiro fazer rastreamento periódico da HAS entre os idosos, tendo em vista a alta prevalência nesta população e necessidade de intervenção precoce.

Referente ao hábito de aferir PA mensalmente, destaca-se que apesar da maioria dos idosos ser aderente, a distância 
entre as fazendas e a unidade de saúde, pode ser um fator limitante. Ademais, a HAS é uma doença, muitas vezes, assintomática o que pode favorecer a menor adesão a terapêutica ${ }^{3}$ e ao controle da PA. Desta forma, os profissionais de saúde podem aproveitar o momento em que o idoso está na unidade para desenvolver ações educativas contribuindo, assim, para melhorar a adesão.

A maioria dos idosos fazia uso de medicamentos e não interromperam o tratamento. Contudo, cabe destacar que a não adesão, geralmente, ocorre pela falta de conhecimento acerca da doença. ${ }^{3}$ Dessa forma, a educação em saúde, com enfoque para as consequências da HAS não controlada e os benefícios a curto, médio e longo prazo do tratamento adequado são sugestões para favorecer a $\operatorname{adesão}^{3} \mathrm{e}$ contribuir para o não abandono da terapêutica.

A redução do excesso de sal na alimentação é uma das estratégias utilizadas para controlar a $\mathrm{PA}^{3}$, a qual deve ser reforçada pelos enfermeiros durante as atividades de educação em saúde.

Referente à QV, o maior escore no domínio relações sociais, em ambos os grupos da presente pesquisa, podem ser justificado devido nessa fase da vida os idosos terem laços familiares e pessoais mais bem determinados ${ }^{15}$, o que possivelmente influencia no maior escore do citado domínio.

O menor escore de QV no domínio meio ambiente pode estar relacionado às maiores limitações encontradas no espaço rural, como a falta de segurança, dificuldade de transporte público, escassez de recreação, aspectos avaliados neste domínio. ${ }^{8}$

Investigação com idosos no Paraná observou que a doença interfere na QV prejudicando as atividades laborais; os sinais e sintomas da doença, muitas vezes, podem impedir a realização das atividades rotineiras de maneira satisfatória ${ }^{16}$, fatores que podem ser relacionados ao menor escore entre aquele com HAS no domínio físico. É necessário que a equipe de saúde incentive a realização de avaliações periódicas a fim de monitorar as condições de saúde e orientar acerca das comorbidades, e assim, contribuir para melhor adaptação à suas condições de saúde e minimizar o impacto sobre a QV.

$$
\text { Concernente aos aspectos }
$$
psicológicos, infere-se que as mudanças decorrentes da doenças possam impactar na QV. No entanto, há de se considerar que o estresse é percebido pelos idosos como 
agente desencadeador da elevação dos níveis pressóricos. ${ }^{16}$ Outro inquérito rural em Minas Gerais observou que os idosos acreditam que, ao manter controle emocional, não necessitam da medicação. $\mathrm{O}$ fato de associar a HAS a sintomas psicológicos como nervosismo, raiva, insônia e tristeza pode dificultar o seu controle, pois os idosos abandonam a medicação por considerar que a doença possui período curto e passageiro sendo ocasionada devido a alguma situação estressora. ${ }^{14}$ Dessa forma, os idosos devem ser investigados quanto suas crenças em relação doença. Soma-se as mudanças de hábitos e estilo de vida devem ser avaliadas pelos profissionais de saúde uma vez que possuem relação com as medidas de tratamento não medicamentoso ${ }^{13}$, e podem melhorar os escores de QV.

Quanto ao domínio meio ambiente, estudo realizado com idosos de uma comunidade pesqueira com doenças crônicas, evidenciou que esses idosos relacionam a QV ao conforto e à acessibilidade ao serviço de saúde. ${ }^{17}$ Deste modo, considerando as possíveis dificuldades de acesso na zona rural, os idosos com HAS podem apresentar maior impacto na segurança física e proteção, cuidados de saúde e sociais: disponibilidade e qualidade entre outros itens, avaliados neste domínio ${ }^{8}$ decorrente do maior número de comorbidades e cronicidade da doença. Outro fator é à distância e o funcionamento dos serviços de saúde, o qual pode ser preocupante aos idosos com doenças crônicas. ${ }^{17}$

A faceta participação social avalia a participação em atividades cotidianas, especialmente na comunidade ${ }^{9}$; e o espaço rural oferece poucas oportunidades de atividades sociais em relação à zona urbana $^{18}$, o que compromete a $\mathrm{QV}$ neste aspecto.

O fato dos idosos com HAS apresentarem menor escore de QV na faceta funcionamento dos sentidos pode estar relacionado, dentre outros fatores, a terapia medicamentosa que pode ocasionar alteração do paladar. $^{3}$ Além disso, as comorbidades podem ocasionar maior impacto nos outros sentidos como visão e audição. Compete ao profissional de saúde, principalmente o enfermeiro, o acompanhamento destes idosos, por meio da investigação sobre o funcionamento sensorial, esclarecimento de dúvidas e a observação do uso de medicamentos e os efeitos colaterais.

Quanto a faceta autonomia, destacase que a doença crônica e outras 
morbidades associadas exigem maior atenção à saúde do idoso, o que pode levar a intervenção da família na tomada de decisões dos idosos e interferir na autonomia destes. ${ }^{19}$ Diante disso, infere-se que a cronicidade da HAS e a presença de comorbidades podem influenciar no menor escore de QV na faceta autonomia.

$\mathrm{O}$ menor escore de $\mathrm{QV}$ na faceta participação social entre os idosos com HAS pode estar relacionado à escassez de atividades no meio rural e também pela cronicidade da doença. Estudo realizado entre idosos com doenças crônicas de uma comunidade pesqueira observou que a não participação nas atividades estavam relacionadas à diminuição de acuidade visual e dificuldade de movimentação. ${ }^{17}$

Dentre os fatores que podem estar influenciando o menor escore de QV na faceta intimidade pode estar o uso de medicamentos para HAS que pode causar disfunção sexual ${ }^{3}$. Além disso, essa faceta avalia as relações sociais e intimas ${ }^{9}$, sendo que em estudo com idosos de uma comunidade pesqueira foi identificado que a desestruturação do suporte social e familiar contribui para o agravo das condições crônica. ${ }^{17} \mathrm{Na}$ consulta de enfermagem junto ao idoso com HAS deve-se indagar sobre as suas relações pessoais para contribuir e incentivá-los a melhorar estes aspectos.

\section{CONCLUSÕES}

Neste estudo, verificou-se que os idosos com HAS apresentaram menores escores de QV na maioria dos domínios e facetas avaliados quando comparados aos que não tinham esta doença. É imprescindível estabelecer ações de saúde que visem minimizar o comprometimento físico e as alterações dos sentidos, além de, incentivarem a autonomia e a participação social por meio de ações na comunidade envolvendo familiares, equipe de saúde e idosos.

Diante disto, considera-se essencial o trabalho do enfermeiro na atenção primária para além do controle e tratamento da HAS. O levantamento detalhado da condição de saúde de cada idoso e das repercussões na sua $\mathrm{QV}$, por meio do processo de enfermagem, subsidiará o julgamento clínico para estabelecer uma assistência de enfermagem particularizada, contextualizada e qualificada.

Ressalta-se como limitações deste estudo o recorte transversal que não permite estabelecer relação causal. 


\section{REFERÊNCIAS}

1. Brasil. Ministério da Saúde. Secretaria de Vigilância em Saúde. Departamento de Análise de Situação de Saúde. Plano de ações estratégicas para o enfrentamento das doenças crônicas não transmissíveis (DCNT) no Brasil 20112022, 2011 [Acesso em 05 de abril de 2016]. Disponível em: http://bvsms.saude.gov.br/bvs/publicacoes/ plano_acoes_enfrent_dent_2011.pdf-

2. Secretaria de Vigilância em Saúde. Vigitel Brasil 2011: vigilância de fatores de risco e proteção para doenças crônicas por inquérito telefônico. Brasília: Ministério da Saúde; 2012. [Acesso em 06 de abril de 2016]. Disponível em http://bvsms.saude.gov.br/bvs/publicacoes/ vigitel_brasil_2011.pdf

3. Sociedade Brasileira de

Cardiologia; Sociedade Brasileira de Hipertensão; Sociedade Brasileira de Nefrologia. VI Diretrizes Brasileiras de Hipertensão. Arq Bras Cardiol. 2010; 95(1 supl.1): 1-51. [Acesso em 10 de maio de 2011]; Disponível em: http://publicacoes.cardiol.br/consenso/201 0/Diretriz_hipertensao_associados.pdf/ 4. Amaral TLM, Amaral CA, Prado PR, Lima NS, Herculano PV, Monteiro GT. Qualidade de vida e morbidades associadas em idosos cadastrados na Estratégia de Saúde da Família do município Senador Guiomard, Acre. Rev. Bras. Geriatr. Gerontol., Rio de Janeiro, 2015; 18(4):797808

5. The WHOQOL Group. The World Health Organization quality of life assessment: position paper from the World Health Organization. Soc Sci Med. 1995; 41(10):1403-9.

6. Bertolucci PH, Bruck SM,
Campacci SR, Juliano YO. O mini-exame do estado mental em uma população geral: impacto da escolaridade. Arq Neuropsiquiatr. 1994; 52(1):1-7.

7. Ramos L, Toniolo N, Cendoroglo $M$ et al. Two-year follow-up study of elderly residents in S. Paulo, Brazil: methodology and preliminary results. Rev Saude Publica. 1998; 32(5):397-407.

8. Fleck MPA, Louzada S, Xavier M, Vieira G, Santos L, Pinzon V. Aplicação da versão em português do instrumento abreviado de avaliação da qualidade de vida "WHOQOL-BREF". Rev Saude Publica. 2000; 34(2):178-83.

9. Fleck MPA, Chachamovich E, Trentini C. Development and validation of the Portuguese version of the WHOQOLOLD module. Rev Saude Publica. 2006; 40(5):785-91.

10. Rigo II, Paskulin LMG, Morais EP. Capacidade funcional de idosos de uma comunidade rural do Rio Grande do Sul. Rev Gaúcha Enferm. 2010; 31(2):254-61. (11)

11. Pereira RJ, Cotta RMM, Franceschini SCC, Ribeiro RCLR, Tinoco AL, Rosado LEFPL, et al. Analysis of the social and health profile of the elderly: the relevance of the Family Health Program. Rev Med Minas Gerais. 2010; 20(1):5-15. (12)

12. Betancourt CL. La transculturación en el cuidado de enfermeira gerontológica comunitária. Revista Cubana de Enfermería. 2012; 28(3):195-208. (13)

13. Pierin AMG, Marroni SN, Taveira LAF, Benseñor IJM. Controle da hipertensão arterial e fatores associados na atenção primária em Unidades Básicas de Saúde localizadas na Região Oeste da cidade de São Paulo. 2011; 16(suppl.1):1389-1400.

14. Soares MM, Silva LOL, Chequer MAA, Rodrigues SM, Dias C. Comportamentos supersticiosos e práticas 
comportamentais inadequadas no tratamento da hipertensão arterial sistêmica. Psic., Saúde \& Doenças. 2014 Jun; 15(2): 524-537.

15. Martins CR, Albuquerque FJB, Gouveia CNN, Rodrigues CFF, Neves MTS. Avaliação da Qualidade de Vida Subjetiva dos Idosos: uma comparação entre os residentes em cidades rurais e urbanas. Estud Interdiscip Envelhec. 2007; 11:135-54.

16. Rissardo LK, Barreto MS, Oliveira AP, Marcon SS, Carreira G. Influência da hipertensão arterial e o tratamento na qualidade de vida de idosos. Rev enferm UFPE. 2012; 6(12):2918-26

17. Meirelles BHS, Arruda C, Simon E, Vieira FMA, Cortezi MDV, Natividade MSL. Condições associadas com à qualidade de vida dos idosos com doenças crônicas. Cogitare Enferm. 2010; 15(3):433-40.

18. Tavares DMS, Paiva MM, Dias FA, Diniz MA, Martins NPF. Características sociodemográficas e qualidade de vida de idosos com hipertensão arterial sistêmica que residem na zona rural: importância do enfermeiro. Rev Latino-Am. Enfermagem. 2013; 21(2):515-22.

19. Tavares DMS, Martins NPF, Diniz MA, Dias FA, Santos NMF. Quality of life of the elderly with hypertension. Rev Enferm UERJ. 2011;19(3):438-44.

Recebido em: 22/09/2015

Aprovado em: 13/07/2016

Publicado em: 31/07/2016 